AUTHOR CORRECTION OPEN

\title{
Author Correction: Mapping mesoscopic phase evolution during E-beam induced transformations via deep learning of atomically resolved images
}

Rama K. Vasudevan (iD ${ }^{1,2}$, Nouamane Laanait ${ }^{3}$, Erik M. Ferragut ${ }^{4,5}$, Kai Wang ${ }^{1,2}$, David B. Geohegan ${ }^{1,2}$, Kai Xiao ${ }^{1,2}$, Maxim Ziatdinov ${ }^{1,2}$, Stephen Jesse ${ }^{1,2}$, Ondrej Dyck ${ }^{1,2}$ and Sergei V. Kalinin ${ }^{1,2}$

npj Computational Materials (2018)4:79; https://doi.org/10.1038/s41524-018-0130-7

Correction to: $n$ pj Computational Materials 2018 https://doi.org/ 10.1038/s41524-018-0086-7, Published online 28 June 2018

In the original version of this Article the term 'rhombohedral' was used incorrectly in place of 'oblique', and the term 'rhombohedral' was used in Supplementary Figure 5 to describe the simulated lattice but we had instead simulated a rectangular centered lattice. All mentions of 'rhombohedral' have been corrected to 'oblique' in the manuscript, Fig. 2 and Fig. 4-6, and Supplementary Information. Similarly, 'centered rectangle' has been corrected to 'rectangular centered lattice' throughout the manuscript to avoid any confusion in terminology. Supplementary Figure 5 was not accurately computed in the original published version, and has now been recomputed to meet the actual definition of an oblique Bravais lattice.

There was also a typographical error in the original version of the Article, where Fig. 3c was referred to instead of Fig. $3 d$ on page 4 of the manuscript.

The above changes have been made to the HTML and PDF versions of the Article.
These errors were alerted to the authors by Prof. M. Nespolo (Université de Lorraine) and Prof. P. Moeck (Portland State University), to whom we are grateful.

\begin{abstract}
Open Access This article is licensed under a Creative Commons Attribution 4.0 International License, which permits use, sharing, adaptation, distribution and reproduction in any medium or format, as long as you give appropriate credit to the original author(s) and the source, provide a link to the Creative Commons license, and indicate if changes were made. The images or other third party material in this article are included in the article's Creative Commons license, unless indicated otherwise in a credit line to the material. If material is not included in the article's Creative Commons license and your intended use is not permitted by statutory regulation or exceeds the permitted use, you will need to obtain permission directly from the copyright holder. To view a copy of this license, visit http://creativecommons. org/licenses/by/4.0/.
\end{abstract}

(c) The Author(s) 2018

\footnotetext{
${ }^{1}$ Center for Nanophase Materials Sciences, Oak Ridge National Laboratory, Oak Ridge, TN 37831, USA; ${ }^{2}$ Institute for Functional Imaging of Materials, Oak Ridge National Laboratory, Oak Ridge, TN 37831, USA; ${ }^{3}$ Computational Sciences and Engineering Division, Oak Ridge National Laboratory, Oak Ridge, TN 37831 , USA and ${ }^{4}$ Quantum Computing Institute, Oak Ridge National Laboratory, Oak Ridge, TN 37831, USA
}

Correspondence: Rama K. Vasudevan (vasudevanrk@ornl.gov)

${ }^{5}$ Present address: UnitedHealth Group, PO Box 1459, Minneapolis 55440 MN, USA

Published online: 18 December 2018 